\title{
El Método Singapur como estrategia didáctica para el fortalecimiento de la competencia de resolución de problemas aditivos en estudiantes de básica primaria
}

\section{The Singapore Method as a didactic strategy for the strengthening of the competence of solving additive problems in elementary school students}

\author{
$a^{*}$ Yeslyn Paola Meneses-Patiño, ${ }^{b}$ Laudid Ardila \\ a* Maestría en Educación, ymeneses178@unab.edu.co, 0000-0002-9134-6140, Colegio Luis Carlos Galán Sarmiento, Cúcuta, \\ Colombia \\ b Maestría en Educación, lardila367@unab.edu.co, 0000-0002-6096-6515, Colegio Luis Carlos Galán Sarmiento, Cúcuta, \\ Colombia
}

Forma de citar: Meneses-Patiño,Y. P. Ardila. L. El Método Singapur como estrategia didáctica para el fortalecimiento de la competencia de resolución de problemas aditivos en estudiantes de básica primaria. Eco Matemático, 10 (1), 28-41

Recibido: 22 agosto 2018

Aceptado: 6 noviembre 2018

\author{
Palabras clave \\ Resolución \\ de problemas \\ aditivos, Estrategia \\ Didáctica, \\ Método Singapur, \\ Competencia \\ matemática.
}

Resumen:La presente investigación titulada "El Método Singapur como Estrategia Didáctica para el fortalecimiento de la Competencia de Resolución de Problemas Aditivos, en estudiantes de segundo y tercer grado de Básica Primaria de la Institución Educativa Colegio Luis Carlos Galán Sarmiento Cúcuta", tuvo como objetivo fortalecer la competencia de resolución de problemas aditivos en estudiantes de los grados segundo y tercero de primaria de la Institución Educativa Colegio Luis Carlos Galán Sarmiento a través de la implementación de una estrategia didáctica basada en el método Singapur. La investigación es de tipo cualitativo debido a que produce datos descriptivos (las propias palabras de las personas, habladas o escritas, y la conducta observable, adicional), se utilizó el diseño de la investigación-acción la cual permite analizar y describir las acciones humanas y las situaciones sociales experimentadas por sus integrantes, tales como problemáticas susceptibles de cambio y aquellas que requieran una respuesta práctica. Para su planteamiento se partió de una revisión bibliográfica, que dio luces al sustento teórico del problema y su posible solución, así como para la implementación de aspectos metodológicos, diseño de instrumentos como el test inicial, el diario pedagógico, la entrevista a otros docentes, estrategias didácticas como referentes para el diseño de las propias y el test final. Paso seguido, se procedió al desarrollo de las estrategias didácticas que generaron aprendizajes significativos en los estudiantes, específicamente en el tema de resolución de problemas aditivos. El impacto en el grupo de estudio fue satisfactorio por cuanto permitió un avance importante en la construcción de conocimiento, capacidad de debatir y argumentar propuestas de solución a los diferentes problemas aditivos planteados en las actividades de clase.

*Autor para correspondencia ymeneses178@unab.edu.co 


\section{Keywords}

Resolution of additive problems, Didactic Strategy, Singapore Method, Mathematical competence.

\section{Introducción}

La presente investigación denominada "El Método Singapur como Estrategia Didáctica para el fortalecimiento de la Competencia de Resolución de Problemas Aditivos, en estudiantes de Básica Primaria" se desarrolló con los estudiantes de segundo y tercer grado de las sedes El Pórtico y San Vicente respectivamente, de la Institución Educativa Colegio Luis Carlos Galán Sarmiento de la ciudad de Cúcuta; esta investigación, coloca de manifiesto las dificultades de los estudiantes en la resolución de problemas aditivos y plantea una propuesta de trabajo basada en el Método Singapur con el objetivo de fortalecer esta competencia en los estudiantes para mejorar el desempeño en las pruebas que miden la calidad educativa.

Inicialmente, se realizó una revisión de las pruebas PISA, donde se destacó que Colombia aún mantiene una brecha en matemáticas respecto a los países miembros de las la OCDE, del mismo modo los resultados para el área de matemáticas de las pruebas saber para el grado tercero de la institución no son muy alentadores, especialmente en la competencia de resolución de problemas, de allí y de la observación del proceso de enseñanza-aprendizaje en el aula, surgió la necesidad de fortalecer dicha competencia matemática, se tuvieron en cuenta, aspectos teóricos tales como competencias matemáticas, resolución de problemas, el método Singapur, estrategias didácticas, entre otras. Se presenta la propuesta pedagógica, con aspectos metodológicos y análisis de su implementación.

Es importante resaltar que la estrategia diseñada e implementada, cumplió en gran parte las expectativas ya que se percibió actitud positiva por parte de los estudiantes en cuanto a la metodología empleada para el desarrollo de las actividades propuestas, debido a qué se abordaron los temas desde la didáctica y esto logró despertar el interés de los niños. 


\section{Materiales y Métodos}

\section{Tipo de Investigación}

Según (Rodriguez, Gil, \& García, 1996) citados por (Galvéz \& Pagés, 2016), la investigación cualitativa estudia la realidad en su contexto natural, con el objetivo de interpretar los fenómenos de acuerdo al significado que tienen para cada persona involucrada; este tipo de investigación implica el uso de una gran cantidad de materiales, como la observación, entrevistas, e imágenes, que describen el quehacer, las diferentes situaciones problemas, así como el significado de éstas en la vida de las personas. De acuerdo a lo anterior, este trabajo de investigación se desarrolla en el marco de la investigación cualitativa, ya que se realiza a través de la implementación de métodos como la observación, entrevistas, notas de campo, fotografías, videos, entre otros.

La propuesta busca fortalecer la competencia de resolución de problemas en estudiantes de los grados segundo y tercero de básica primaria de la Institución Educativa Colegio Luis Carlos Galán Sarmiento a través de diversas estrategias pedagógicas, se llevó a cabo por medio del enfoque cualitativo, bajo el diseño investigación acción educativa. Este tipo de investigación se realiza para generar cambios en el ámbito educativo específicamente en la enseñanza y el aprendizaje de los estudiantes. (Kemmis \& McTaggart, 1988) citados por (Morales, 2010), s.f) señalan que la investigación- acción es:

Un proceso que (i) Se construye desde y para la práctica, (ii) pretende mejorar la práctica a través de su trasformación, al mismo tiempo que procura comprenderla, (iii) demanda la participación de los sujetos en la mejora de sus propias prácticas, (iv) exige una actuación grupal por la que los sujetos implicados colaboran coordinadamente en todas las fases del proceso de investigación, (v) implica la realización de análisis crítico de las situaciones y (vi) se configura como una espiral de ciclos de planificación, acción, observación y reflexión.(p.2).
(Elliott, 1978) citado por (Rodríguez \& Yáñez, 2012), señala que en la investigación acción:

Se investigan acciones y situaciones en las que están implicados los docentes, situaciones que para ellos son problemáticas, que pueden ser modificadas y que, por lo tanto, admiten una respuesta práctica. No se trata de problemas teóricos, ni de cuestiones que sean de interés exclusivo para los académicos o expertos; puede haber coincidencia, pero es imprescindible que el objeto de la exploración sea un problema vivido como tal por los profesores.

Con referencia a lo anterior, esta investigación estuvo orientada en la búsqueda de soluciones a las dificultades que presentan los estudiantes, en la comprensión y solución a situaciones problemas propuestos en el aula de clase de acuerdo al contexto donde se vivencia la situación planteada.

\section{Proceso de investigación}

Para la puesta en marcha del proceso de investigación acción, el desarrollo de esta se dividió en tres fases: Revisión de información y diagnóstico, diseño e implementación, y evaluación, con el propósito de alcanzar el objetivo general, fortaleciendo la competencia de resolución de problemas aditivos en estudiantes de los grados segundo y tercero de primaria de la Institución Educativa Colegio Luis Carlos Galán Sarmiento para potenciar su desarrollo académico y personal.

\section{Fase I. Revisión de información y diagnóstico}

Se parte de la recopilación y revisión de información secundaria (durante el primer $\mathrm{y}$ segundo semestre de 2016), relacionada con resultados en pruebas PISA de Colombia en el año 2015 y pruebas saber de grado tercero en el área de matemáticas para los años 2013-2016 de la I.E, con el fin de determinar el nivel de desempeño de los estudiantes; además de la revisión de diferentes investigaciones (general y maestría) que sirvieron como sustento teórico para el diseño y desarrollo de nuestra propuesta. A continuación, se procedió con 
el diseño y la realización de una prueba diagnóstica, con el fin de identificar las dificultades que presentan los estudiantes en la resolución de aditivos, y una entrevista a docentes de grado segundo y tercero que al momento de aplicar la prueba, para conocer como imparten ellas la enseñanza de la temática de resolución de problemas aditivos, y de esta manera establecer el camino a seguir; dicho diagnóstico se realizó a finales del año 2016, y las intervenciones, donde se desarrollaron las actividades diseñadas en la propuesta se iniciaron con los mismos grupos en el primer semestre de 2017, pero se vieron afectadas por el cese de clases debido al paro nacional de maestros, por lo que las intervenciones se extendieron hasta el segundo semestre de 2017.

\section{Fase II. Diseño}

Partiendo de los resultados se busca una solución a las dificultades identificadas, para ello se realiza el diseño de una propuesta, integrando diferentes estrategias en una unidad didáctica, orientada a los niños de segundo y tercero, con base a las dificultades observadas, especialmente, la extracción de información de un problema, la construcción de los conceptos de adición y sustracción, y problemas de estructura aditiva. Se desarrolla la unidad didáctica, que consta a su vez, de tres guías, y cada una de éstas, de varias sesiones: cada guía se basa en el Método de Singapur (Enfoque CPA: concreto, pictórico y simbólico), integrando el uso de material concreto, la lúdica matemática, los juegos matemáticos y la técnica del aprendizaje colaborativo, que permiten el fortalecimiento de la competencia de resolución de problemas aditivos.

\section{Fase III. Implementación-Evaluación}

Las guías de la unidad didáctica, se aplican a una muestra de los grados segundo y tercero de dos sedes de la (I.E), para construir aprendizaje significativo a partir de la comprensión de las situaciones problemas matemáticas propuestas. Para la fase de implementación, se conformaron grupos con los niños para fortalecer el aprendizaje colaborativo, de igual modo, se establecieron acuerdos de trabajo; se utilizaron diferentes materiales, entre ellos, tapitas, chaquiras, botones, etc., para el desarrollo de las actividades iniciales, con el fin de motivar y despertar el interés de los estudiantes; se iniciaba con estas actividades, para continuar lluvias de ideas, para la construcción grupal de conceptos, después, se buscaba afianzar el conocimiento construido con el planteamiento de diferentes problemas aditivos, y se finalizaba con una actividad de retroalimentación. Por último, se valoró la pertinencia y eficacia de la estrategia didáctica implementada, a través de la aplicación de otra prueba, para analizar de nuevo el desempeño de los estudiantes, con el fin de determinar la efectividad de las actividades propuestas en la estrategia, con el fin de replicar aquellas que fueron más efectivas en la I.E.

\section{Categorías de análisis}

Las categorías de análisis que se tienen en cuenta en la presente investigación se ilustran en la Tabla I.

Tabla I. Categorías de análisis y subcategorías

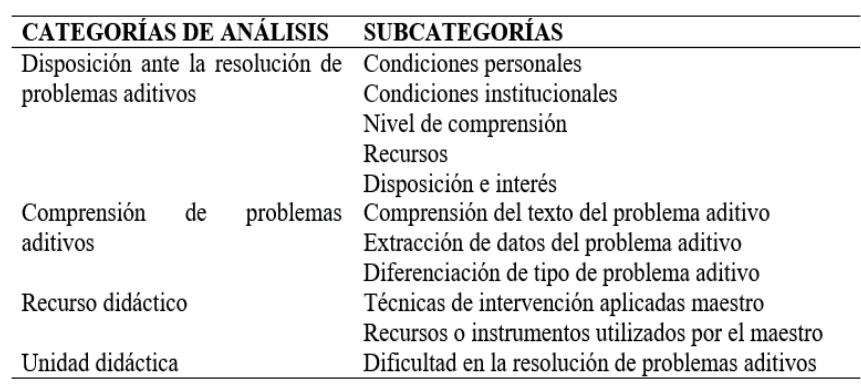

\section{Desarrollo de la estrategia didáctica}

La unidad didáctica dirigida al grado segundo, se desarrolló, a través de tres guías didácticas, cada guía didáctica a su vez, estuvo dividida en sesiones, la primera guía denominada "preliminares", la segunda guía, "problemas aditivos”, y la última guía "aplicaciones", de dos sesiones cada una. La unidad didáctica dirigida al grado tercero, se desarrolló, a través de tres guías didácticas, cada guía didáctica a su vez, estuvo dividida en sesiones, la primera guía denominada "preliminares", constó de dos sesiones; la segunda guía, "problemas aditivos", constó de 
tres sesiones; y la última guía "aplicaciones", de tres sesiones. Cada sesión se encuentra dividida en tres fases, primero, la fase inicial, donde se introduce cada tema, después, la fase de desarrollo, donde a través de situaciones planteadas se construyen conceptos, y por último, la fase de consolidación, donde se refuerzan los conceptos construidos y los saberes adquiridos. Como se ha mencionado de forma anterior, estas unidades didácticas están basadas en el Método Singapur, por lo que se trata concreto (material), lo pictórico (imágenes) y lo simbólico (situaciones).

\section{Resultados y discusión}

\section{Test inicial}

Según los resultados del test inicial, se puede inferir, que tanto los estudiantes de segundo como los de tercer grado presentan mayor dificultad para responder correctamente los ítems abiertos con respuesta corta que los ítems cerrados con opción múltiple y única respuesta, además, tanto los estudiantes de segundo como de tercer grado tienen dificultad en los conceptos de disminuir y aumentar. Así mismo, la mayoría de los estudiantes, tuvo dificultades en el momento de extraer los datos de un problema matemático, lo que dificultó su análisis y solución.

Otro aspecto relacionado con las preguntas que tienen un porcentaje de desacierto mayor a la mitad de los estudiantes, es el hecho de que éstas están asociadas a la comprensión del problema, es decir, los niños tienen problemas para comprender, qué dice el problema, qué me está pidiendo el problema, qué proceso u operación debo realizar para resolver el problema. Aunque se observa que la mayoría de estudiantes acertaron algunas preguntas, cuyos problemas planteados correspondan a situaciones similares de otros que no acertaron o donde se deba hacer el mismo tipo de operación (adición o sustracción); algunos de estos conllevan más dificultades que otros para la resolución por parte de los estudiantes, esencialmente, cómo se mencionó en el numeral anterior, porque no entienden lo que dice el problema, y cómo no lo entienden, pues no lo pueden resolver, así ellos conozcan las operaciones aditivas básicas.

\section{Análisis de implementación de la propuesta de intervención-grado segundo}

Considerando relevante que el docente debe mostrar un su quehacer pedagógico, una planeación bien estructurada, métodos e instrumentos necesarios para que los estudiantes comprendan mejor los contenidos y tenerles cuenta los pre-saberes y así lograr mejores resultados en este caso en el área de matemáticas.

De igual manera la metodología activa aplicada, combinó, el método Singapur y el trabajo cooperativo, como propuesta para mejorar el desarrollo de las competencias matemática en resolución de problemas en el grado segundo. Pretende ser una respuesta al empeño por cambiar la manera de aprender en el aula de clase e intentar tomar contenidos y transformarlos para qué la recepción por parte del estudiante sea más significativa, claro está con el acompañamiento del docente los estudiantes reflejaron cambio de actitud, frente a las matemáticas donde estuvieron interactuando, interesados y motivados por trabajar con material didáctico, ya que en otras oportunidades no se estaba trabajando con material concreto, sobre todo habría una estudiante que desde el año pasado expresaba apatía y desinterés por la matemáticas y en el trascurso de mitad de este año, fue notorio el cambio, convirtiéndose en una de la líderes de su grupo y motivando a los demás a trabajar ; a la hora de desarrollar los problemas siempre querían escribir las respuestas en la guía, demostraron habilidades a la hora de dar las respuestas, todos se mostraron participativos, manifestaron alegría y con deseos de ser escuchados y se exigían por ser los primeros en su grupo (Ver Figura 1) para obtener su máxima puntuación en la escalera de la metacognición. 


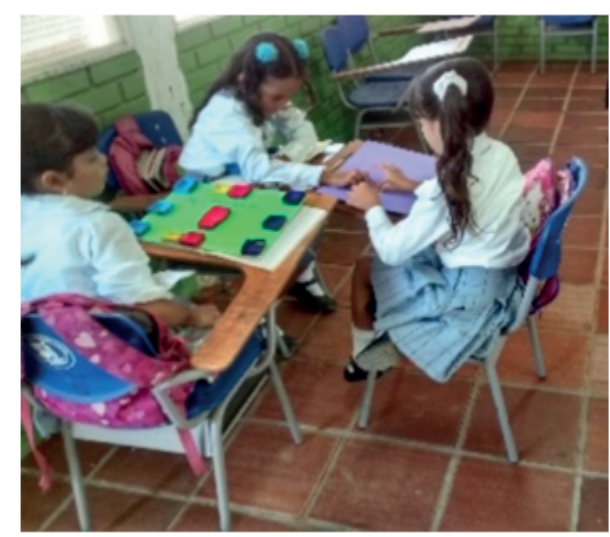

Figura 1. Trabajo en equipo grado segundo

Un estudiante que se mostró apático en las actividades realizadas durante las intervenciones, ya que venía presentados inconvenientes de comportamiento inadecuado dentro y fuera del aula, sin embargo, en las últimas dos intervenciones se le vio desarrollando los problemas de composición y de trasformación en su guía y compartiendo material con los compañeros, se logró una buena relación con el estudiante y con los demás compañeros, en un ambiente de dialogo, escucha, respeto, y de empatía.

La elaboración de las tres cajas con cartón y pintadas con témperas (caja Mackinder, caja de liro de combinación y de trasformación), han sido una experiencia exitosa en el aula, porque los estudiantes demostraron sus habilidades artísticas (Ver Figura 2), compartieron en equipo, y su utilización fueron de gran ayuda para el desarrollo de los problemas aditivos, junto con los billetes, monedas didácticas y demás materiales proporcionados.

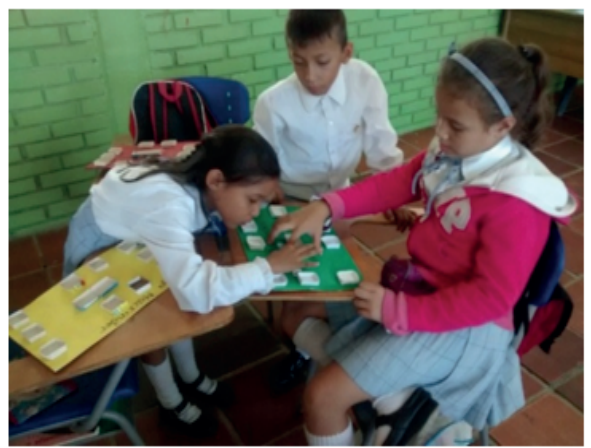

Figura 2. Estudiantes de segundo utilizando caja Mackinder
Por último, es de especificar que las guías aplicadas se trabajaron tanto en el grado segundo "A" como en grado tercero "A", teniendo claro, que en segundo se inicia con los problemas aditivos de composición y de transformación, en concordancia con lo sugerido en los derechos básicos de aprendizaje y en tercero se refuerzan estos y se trabajan también los problemas de comparación, en el cual se desarrollaron dos secciones más (Ver Figura 3).

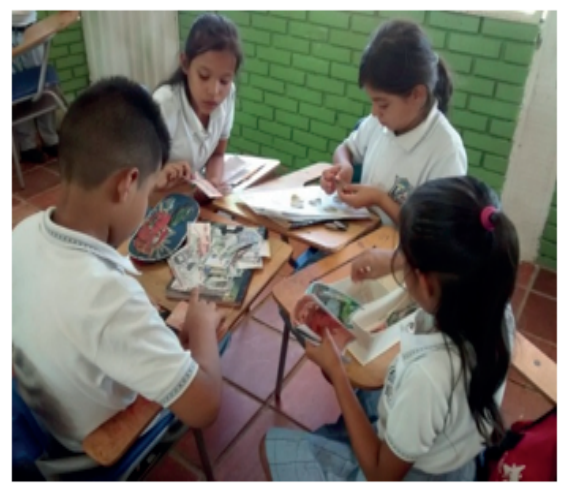

Figura 3. Practicando problemas aditivos

\section{Análisis de implementación de la propuesta de intervención-grado tercero}

Para conseguir resultados satisfactorios en las diferentes actividades a implementar, es fundamental que el maestro este bien preparado en su disciplina y que además seleccione los recursos didácticos y los métodos acordes a los procesos que va a orientar, de ahí la importancia de realizar una buena planeación teniendo en cuenta todo lo anteriormente mencionado. Nuestra labor pedagógica debe estar bien planeada y organizada, de tal manera que podamos impartir confianza en nuestros niños y les podamos brindar una buena orientación en la construcción de su conocimiento. 


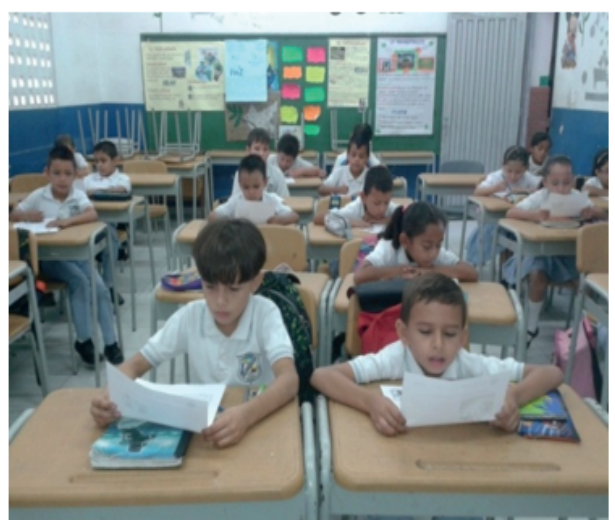

Figura 4. Estudiantes de grado tercero resolviendo el test inicial

Teniendo en cuenta lo anterior, en el desarrollo de la propuesta se plantearon actividades importantes basadas en el método Singapur y encaminadas en el fortalecimiento de la competencia de resolución de problemas matemáticos aditivos, con la intención de que los estudiantes superaran las dificultades identificadas (Ver Figura 4) y desarrollaran habilidades y aptitudes para mejorar el nivel de comprensión de los textos de los diferentes tipos de problemas aditivos, favoreciendo en ellos el trabajo cooperativo en el aula de clase, la interacción entre ellos mismos, con la intención de equilibrar sus conocimientos y que de esta forma adquirieran confianza y seguridad.

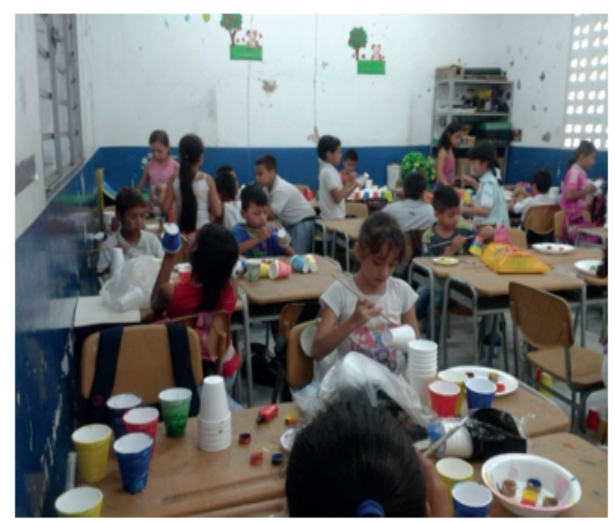

Figura 5. Estudiantes de grado tercero construyendo la caja Mackinder
De igual forma esta propuesta fue de gran importancia, debido a que el estudiante aprendió de una manera diferente, ya que el uso de los recursos didácticos (Ver Figura 5), como lo fue el material concreto estructurado y no estructurado y el luego realizar la representación pictórica y simbólica de lo que se había representado con estos recursos didácticos, despertó una gran motivación en el estudiante por aprender a solucionar los diferentes tipos de problemas matemáticos aditivos y los niños que mostraban apatía hacia el área de matemáticas, se mostraron la mayor parte del tiempo dispuestos a aprender y participar en las diferentes actividades, contribuyendo con ello al logro de objetivos plasmados en las diferentes estrategias didácticas desarrolladas, todo lo anterior hace que el maestro se enamore más de su práctica y se sienta motivado y comprometido con la misma.

Por otra parte, la metodología emprendida en esta investigación permitió conocer la forma como se debía organizar el trabajo, ya qué se utilizó la investigación acción, la cual implica observar, registrar, recopilar y llevar un diario, donde se deben registrar las reflexiones de nuestra praxis y de los diferentes momentos que se viven en el aula clase junto con los estudiantes.

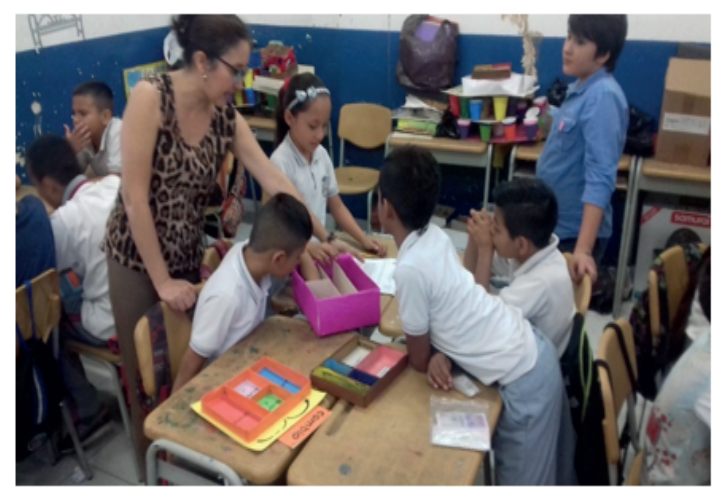

Figura 6. Niños de tercer grado trabajando los distintos tipos de problemas aditivos 
Tabla II. Resultados test final segundo

\begin{tabular}{|c|c|c|c|c|c|c|c|c|}
\hline ITEM & PREGUNTA & $\begin{array}{c}\text { TIPO DE } \\
\text { PREGUNTA }\end{array}$ & $\begin{array}{l}\text { COMPETENCIA } \\
\text { MATEMATICA }\end{array}$ & $\begin{array}{c}\text { NIVEL DE } \\
\text { COMPLEJIDAD }\end{array}$ & ACIERTO & $\%$ & DESCACIERTO & $\%$ \\
\hline \multirow{5}{*}{$\begin{array}{c}\text { CERRADO- } \\
\text { OPCIÓN } \\
\text { MÚLLTPLE } \\
\text { UUNICA } \\
\text { RESPUESTA }\end{array}$} & 1 & $\begin{array}{c}\text { Operación } \\
\text { básica-aditiva }\end{array}$ & $\begin{array}{l}\text { Tratamiento y } \\
\text { resolución de } \\
\text { problemas }\end{array}$ & ALTO & 6 & 42,9 & 8 & 57,1 \\
\hline & 2 & $\begin{array}{c}\text { Operación } \\
\text { básica-aditiva }\end{array}$ & $\begin{array}{l}\text { Tratamiento y } \\
\text { resolución de } \\
\text { problemas }\end{array}$ & MEDIO & 9 & 64,3 & 5 & 35,7 \\
\hline & 3 & $\begin{array}{c}\text { Operación } \\
\text { básica-aditiva }\end{array}$ & $\begin{array}{l}\text { Tratamiento y } \\
\text { resolución de } \\
\text { problemas }\end{array}$ & MEDIO & 8 & 57,1 & 6 & 42,9 \\
\hline & 4 & $\begin{array}{c}\text { Operación } \\
\text { básica-aditiva }\end{array}$ & $\begin{array}{l}\text { Tratamiento y } \\
\text { resolución de } \\
\text { problemas }\end{array}$ & ALTO & 10 & 71,4 & 14 & 28,6 \\
\hline & 5 & $\begin{array}{c}\text { Operación } \\
\text { básica-aditiva }\end{array}$ & $\begin{array}{l}\text { Tratamiento y } \\
\text { resolución de } \\
\text { problemas }\end{array}$ & MEDIO & 10 & 71,4 & 14 & 28,6 \\
\hline
\end{tabular}

Las estrategias didácticas que se aplicaron tuvieron una buena aceptación por parte de los estudiantes, pues se logró llevar a cabo todas las actividades que se habían planeado y esto permitió que los estudiantes se interesaran más por aprender y evaluar su propio aprendizaje, a la vez que interactuaban con sus compañeros en los diferentes grupos de trabajo cooperativo (Ver Figura 6). Del mismo modo, es necesario mencionar las tres fases como se planearon

las actividades: la fase inicial, es el momento donde el maestro articula el conocimiento y explora los saberes previos de los estudiantes a través de preguntas relacionadas con la temática a trabajar, en la fase de desarrollo de la actividad y las estrategias, es donde se aplican las diferentes estrategias a trabajar y se desarrolla la temática. Y finalmente la fase de consolidación de los aprendizajes, es donde se refuerza y evalúa lo aprendido por los estudiantes, para ratificar los conocimientos adquiridos por los mismos.

\section{Test Final}

\section{Resultados del test final-grado segundo}

Según los resultados del test final (Ver Tabla II) se puede decir que:

El error más frecuente se presentó en la pregunta 1 donde el $57 \%$ de los estudiantes erraron debido a que les falto más releer el problema de trasformación para comprenderlo, y a que no recordaron la palabra clave (menos), que indica que debían que realizar una sustracción, En la pregunta 2 , el 64,3 \% respondió con mayor proporción de acierto, esto significa aplicaron bien la palabra clave, y la operación de adición. En la pregunta 3, el 57,1\% acertó que operación debían que tener en cuenta con relación al problema dado, los demás no relacionaron el problema con la operación. Se resalta que la mayoría respondieron bien la pregunta 4 , con un porcentaje del $71,4 \%$, donde combinando billetes y monedas obtenían el resultado del dinero. A igual que la pregunta 5 acertaron con el mismo porcentaje, pero aplicando la adición en la resolución del problema. Se observó que la mayoría de estudiantes del grado tercero contestó acertadamente más de tres de las cinco preguntas del test, lo que demuestra el fortalecimiento de su competencia de resolución de problemas aditivos.

\begin{tabular}{|c|c|c|c|c|c|c|c|c|}
\hline ITEM & PREGUNTA & $\begin{array}{c}\text { TIPODE } \\
\text { PREGUNTA }\end{array}$ & $\begin{array}{l}\text { COMPETENCIA } \\
\text { MATEMATICA }\end{array}$ & $\begin{array}{c}\text { NIVEL DE } \\
\text { COMPLEIDAD }\end{array}$ & ACIERTO & $\%$ & DESCACIERTO & $\%$ \\
\hline \multirow{5}{*}{$\begin{array}{l}\text { CERRADO- } \\
\text { OPCIÓN } \\
\text { MÚLTIPLE } \\
\text { ÚNICA } \\
\text { RESPUESTA }\end{array}$} & 1 & $\begin{array}{l}\text { Operación básica- } \\
\text { aditiva }\end{array}$ & $\begin{array}{c}\text { Tratamiento y } \\
\text { resolución de problemas }\end{array}$ & MEDIO & 36 & 85,71 & 6 & 14,29 \\
\hline & 2 & $\begin{array}{c}\text { Operación bảicica- } \\
\text { aditiva }\end{array}$ & $\begin{array}{l}\text { Tratamiento y } \\
\text { resolución de problemas }\end{array}$ & MEDIO & 37 & 88,10 & 5 & 11,90 \\
\hline & 3 & $\begin{array}{c}\text { Operación básica- } \\
\text { aditiva }\end{array}$ & $\begin{array}{c}\text { Tratamiento y } \\
\text { resolución de problemas }\end{array}$ & ALTO & 13 & 33,95 & 29 & 69,05 \\
\hline & 4 & $\begin{array}{c}\text { Operaciön bảica- } \\
\text { aditiva }\end{array}$ & $\begin{array}{c}\text { Tratamiento y } \\
\text { resolución de problemas }\end{array}$ & ALTO & 36 & 85,71 & 6 & 14,29 \\
\hline & 5 & $\begin{array}{c}\text { Operación basica- } \\
\text { aditiva }\end{array}$ & $\begin{array}{c}\text { Tratamiento y } \\
\text { resolución de problemas }\end{array}$ & ALTO & 22 & 52,38 & 20 & 47,62 \\
\hline
\end{tabular}

\section{Resultados del test final-grado tercero}

Según los resultados del test final (Ver Tabla III) se puede decir que:

La mayoría de los estudiantes de tercer grado respondió acertadamente casi todas las preguntas con excepción de la pregunta 3 , las preguntas de nivel de complejidad fueron respondidas acertadamente por la mayoría de los estudiantes de tercer grado. Aunque hay tres preguntas con nivel dificultad alto, la mayoría de los estudiantes de tercer grado tuvo dificultad para responder la pregunta 3, debido probablemente a que requiere mayor análisis. Se observó que la mayoría de estudiantes del grado tercero contestó acertadamente más de tres de las 
cinco preguntas del test, lo que demuestra el fortalecimiento de su competencia de resolución de problemas aditivos.

\section{Triangulación de la información}

Teniendo en cuenta las categorías establecidas para esta investigación, se presenta la relación y el análisis a través del desarrollo de la propuesta (Ver Tabla IV).

Tabla IV. Triangulación de la información

\begin{tabular}{lcll}
\hline $\begin{array}{l}\text { CATEGORÍAS } \\
\text { ANÁLISIS }\end{array}$ & DE & SUBCATEGORÍAS & RELACIÓN Y ANÁLISIS \\
\hline $\begin{array}{l}\text { Disposición ante } \\
\text { resolución }\end{array}$ & la & Condiciones personales & La mayoría de los niños, antes del desarrollo de la \\
problemas aditivos & & & $\begin{array}{l}\text { propuesta, sentian frustración ante la resolución de } \\
\text { un problema aditivo planteado, debido a su falta } \\
\text { de comprensión, con el desarrollo de las }\end{array}$ \\
& & & $\begin{array}{l}\text { actividades, los niños se sintieron más } \\
\text { empoderados y seguros para dar solución a } \\
\text { problemas aditivos. }\end{array}$
\end{tabular}

Anterior al desarrollo de la propuesta, pocas se trabaja en el aula con material concreto o en grupo, estas condiciones cambiaron durante el desarrollo

Nivel de comprensión de la propuesta, lo que influyó en que el ambiente escolar fuera más ameno y el espacio fuera más confortable debido a la disposición de las mesas y el mayor espacio disponible en el aula.

Recursos

Muchos niños, tenian inicialmente dificultades para la comprensión de los problemas aditivos y la extracción de los datos, pero durante el transcurso del desarrollo de las actividades, los niños adquirieron habilidades, que les permitieron superan dichas dificultades.

Disposición e interés

Como se mencionó de forma anterior, inicialmente se trabajaba poco con el uso de material concreto, con el desarrollo de las intervenciones propuestas, las guías, el uso de elementos como tapitas, chaquiras, etc., fortaleció la comprensión de los problemas aditivos por parte de los estudiantes.

La mayoría de los niños, antes del desarrollo de la propuesta mostraban poco interés o ánimo por resolver problemas aditivos, pero con el uso del material concreto y el aprendizaje colaborativo, tenían mayor disposición e incluso se divertían mientras daban solución a los problemas aditivos planteados.

Comprensión de Comprensión del texto del problemas aditivos problema aditivo

A1 inicio, los estudiantes tenían dificultades para comprender el texto del problema aditivo (qué decía, que operación aditiva realizar, cómo dar respuesta), después del desarrollo de la propuesta, los estudiantes desarrollaron una mayor capacidad de análisis que les permitió comprender el texto de Extracción de datos del cada problema aditivo planteado. problema aditivo

Antes los niños tenían dificultades para identificar los datos claves para dar solución a problemas aditivos, después de la implementación de la propuesta, la mayoría de los estudiantes pudo Diferenciación de tipo de superar esta dificultad, y reconocían más problema aditivo fácilmente los datos claves. 


\begin{tabular}{|c|c|c|}
\hline & & $\begin{array}{l}\text { Anteriormente, los estudiantes presentaban } \\
\text { problemas para reconocer que tipo de problema } \\
\text { aditivo les planteaban y qué operación aditiva } \\
\text { debían utilizar para encontrar las solución, } \\
\text { posterior al desarrollo de las actividades de } \\
\text { intervención, la mayoría de los estudiantes pudo } \\
\text { superar fácilmente este inconveniente y dar } \\
\text { solución al problema aditivo. }\end{array}$ \\
\hline \multirow[t]{2}{*}{ Recurso didáctico } & $\begin{array}{l}\text { Técnicas de intervención } \\
\text { aplicadas maestro }\end{array}$ & $\begin{array}{l}\text { Anteriormente, las clases de matemáticas se } \\
\text { desarrollaban más tradicionalmente, con la } \\
\text { implementación de esta estrategia basada en el } \\
\text { método Singapur, se desarrollaron las actividades } \\
\text { con base en lo concreto, pictórico y simbólico, } \\
\text { además del aprendizaje colaborativo; permitió que } \\
\text { la mayoría de los estudiantes fortalecieran su } \\
\text { competencia de resolución de problemas. }\end{array}$ \\
\hline & & $\begin{array}{l}\text { Antes, las clases donde se trabajan las problemas } \\
\text { aditivos eran más convencionales, con el uso de } \\
\text { las guías diseñadas, el material concreto } \\
\text { empleado, la competencia de resolución de } \\
\text { problemas aditivos se vio fortalecida en la } \\
\text { mayoría de estudiantes y su disposición ante éstos } \\
\text { mejoró. }\end{array}$ \\
\hline Unidad didáctica & $\begin{array}{l}\text { Dificultad en la resolución de } \\
\text { problemas aditivos }\end{array}$ & $\begin{array}{l}\text { Inicialmente, se observaba que los estudiantes } \\
\text { presentaban gran dificultad a la hora de resolver } \\
\text { problemas aditivos debido a distintas situaciones } \\
\text { expuestas con anterioridad, después de la } \\
\text { implementación de esta unidad didáctica basada } \\
\text { en el método Singapur, la mayoría de estudiantes } \\
\text { superó dichas dificultades y lograron resolver los } \\
\text { problemas aditivos planteados acertadamente. }\end{array}$ \\
\hline
\end{tabular}

\section{Conclusiones}

Una vez culminada la investigación y llevado a cabo la interpretación de cada uno de los objetivos específicos que se formularon como punto de inicio para lograr el objetivo general, se concluyó lo siguiente: esta investigación permitió el uso y la aplicación de estrategias didácticas fundamentadas en el método Singapur, cuya finalidad era fortalecer la competencia de resolución de problemas matemáticos aditivos en los estudiantes de segundo y tercer grado.

Las estrategias trabajadas se pueden pensar como un instrumento útil para mejorar el nivel de comprensión textual de los problemas aditivos en los estudiantes, ya que permite que el estudiante interactúe en grupos de trabajo cooperativo, donde el niño aprende mediante la representación concreta, pictórica y simbólica, a encontrar la solución de los diferentes tipos de problemas aditivos y sus variaciones, lo cual le permite mejorar sus propios procesos cognitivos, porque no solo se trata de resolver problemas matemáticos, sino de aspectos importantes de su propio aprendizaje. Los resultados alcanzados señalan que se pueden lograr grandes cambios a partir de la implementación de este tipo de estrategias didácticas, ya que para los estudiantes es más fácil aprender a solucionar este tipo problemas matemáticos aditivos a partir de la aplicación del método Singapur. Así mismo, es fundamental el dominio de estas estrategias didácticas por parte del maestro, para que pueda lograr grandes cambios en el desempeño académico de los estudiantes. Cabe resaltar que las estrategias de aprendizaje son fundamentales para mejorar los procesos académicos de los estudiantes y que es un compromiso del maestro mantenerse actualizado, tanto en su disciplina 
como en el manejo de instrumentos actualizados y de esta manera desarrollar el pensamiento de los estudiantes.

Se observó que a pesar de que los estudiantes mejoraron el nivel de comprensión de los textos de problemas matemáticos aditivos, gracias a todas las actividades planeadas en la unidad didáctica, los tipos de problemas que más se les facilito resolver fueron los problemas aditivos de composición y cambio o transformación, tal vez porque fueron más fáciles de representar y comprender para los estudiantes. Sin embargo, en la evaluación final se evidenciaron avances en los estudiantes, porque demostraron que identificaban el tipo de problema aditivo a resolver y la operación necesaria para poder solucionarlo.

Además, se puede mencionar que las diferentes actividades plasmadas en la unidad didáctica, que se diseñó para fortalecer la competencia de resolución de problemas aditivos fundamentada en el método Singapur, fueron significativas en cuanto a la disposición de los estudiantes, comparada con la actitud de pereza y apatía que presentaban los niños antes de su aplicación; la aplicación de estas estrategias despertó el interés y la motivación de los niños hacia la comprensión y solución de estos tipos de problemas aditivos.

Por otro lado, la mayoría de estos niños, son niños solos, porque sus padres trabajan todo el día, algunos son hijos de familias disfuncionales, otros solo viven con alguno de sus padres, en fin, viven en un contexto de descomposición familiar complicado, lo cual hace que este niño no se sienta motivados e interesados por aprender, pues ellos no entienden la importancia de esto, para desenvolverse en la vida cotidiana y para enfrentarse al futuro. Por este motivo, es importante aplicar este tipo de estrategias que conlleven a la motivación de los estudiantes y faciliten su aprendizaje y a la vez, los conduzcan a obtener grandes logros que les permitan desarrollar sentimientos de seguridad y confianza en sus capacidades, se observó que la implementación de estas estrategias permiten que los estudiantes desarrollen habilidades que les facilita la comprensión de los diferentes tipos de textos de los problemas aditivos, lo cual influye en el mejoramiento de los procesos académicos en el área de las matemáticas.

Se observó que las palabras claves, y el releer el problema, ayudó a mejorar notablemente en la resolución de problemas aditivos, al igual que la aplicación de las diferentes actividades en la unidad didáctica, fue de suma importancia, frente a una desmotivación por parte de varios estudiantes; lo cual la aplicación de la estrategia didáctica, motivo el interés por las matemáticas, donde se refleja que esta estrategia elegida fue adecuada ya que se incentivó la confianza en sus propias capacidades y el desarrollo el pensamiento lógico matemático.

Por consiguiente, se considera esta estrategia como una herramienta fundamental donde se promueve la comunicación y espacios de trabajo cooperativo, en busca de mejores resultados tanto individuales como grupales y a la vez indagan, descubran y aplican conceptos que le ayudan a comprender y resolver los problemas matemáticos de una manera más vivencial, contextualizado a la realidad.

\section{Recomendaciones}

Es importante tener en cuenta el nivel de comprensión de los estudiantes en los textos de los diferentes tipos de problemas aditivos, ya que, sin ello, difícilmente el estudiante podrá encontrar el camino para dar solución al problema matemático planteado, además es recomendable el uso de recursos didácticos que le permitan al estudiante representar, visualizar y de este modo comprender con facilidad el problema matemático propuesto.

Esta propuesta pedagógica puede ser de gran utilidad para otros docentes que quieran fortalecer la comprensión y resolución de problemas matemáticos 
aditivos en los estudiantes. Ya que en ella se trabajan actividades que permiten que los estudiantes se involucren con el área de las matemáticas de una manera más didáctica y vivencial, pues también se trabajan problemas teniendo en cuenta el contexto de los niños. Pero a la vez para aquellos docentes que no conocen esta estrategia, se sugiere una capacitación pedagógica en cuanto a su fundamentación $\mathrm{y}$ experiencias significativas.

Es necesario que en la institución educativa se implementen propuestas de este tipo en los diferentes grados de escolaridad, para que se fortalezcan las competencias matemáticas en los estudiantes y a la vez se mejoren los procesos metacognitivos de los mismos. Pues la búsqueda de la calidad educativa es un compromiso de toda la comunidad educativa en general.

Otro aspecto relevante es la actualización y preparación de los maestros, para poder brindar mejores métodos y estrategias a los estudiantes y de esta manera fortalecer las prácticas pedagógicas, pues en este mundo tan globalizado, donde cada día surgen nuevos métodos y estrategias, podemos convertirnos en personas poco aptas para impartir conocimiento.

\section{Referencias}

Ausubel, D. (1983). Teoría del aprendizaje significativo. Fascículos de CEIF, 1 .

Barón, L., \& Muller, O. (2014). La Teoría Lingüística de Noam Chomsky: del Inicio a la Actualidad. Lenguaje-Univalle, 26.

Blanco, \& Cárdenas. (2013). La Resolución de Problemas como contenido en el Currí-culo de Matemáticas de Primaria y Secundaria. Campo Abierto, 20.

Bruner, J. (1960). El Proceso de la Educación. México: Editorial Hispano Americana.

Bueno, D. (2012). Propuesta metodológica para mejorar la interpretación, análisis y solución de ejercicios y problemas matemáticos en los estudiantes de quinto grado de la Institución
Educativa Alejandro Vélez Barrientos. Bogotá: Universidad Nacional de Colombia.

Calderón, P. (2014). Percepciones de los y las docentes del primer ciclo básico, de la implementación del Método Singapur en el Colegio Mario Bertero Cevasco de la comuna de Isla de Maipo. Chile: Universidad de Chile.

Calzadilla, M. (2010). Aprendizaje colaborativo y tecnologías de la información y la comunicación. Revista Iberoamericana de Educación.

Campos, M. \&. (2004). Estrategia didáctica para la construcción de conocimiento. México: UNAM.

Campos, M. (2010). Comunidades educativas de construcción de conocimiento y nuevas tecnologías: elementos teóricos para su análisis. Sinéctica, 17.

Cano, A. (2015). Avanzando en la investigaciónen didáctica de la traducción:vías metodológicas. Opción, 16.

Cantoral, R. (2013). Teoría socioepistemológica de la matemática educativa. Barcelona: Gedisa.

Cantoral, R., Farfán, R., Lezama, J., \& Martínez, G. (2006). Socioepistemología y representación: algunos ejemplos. RELIME. Revista latinoamericana de investigación en matemática educativa, 9.

Cárdenas, A. (2011). Piaget: lenguaje, conocimiento y Educación. Revista Colommbiana de Educación, 21.

Chomsky, N. (1989). El conocimiento del lenguaje, su naturaleza, origen y uso. Madrid: Alianza Editorial.

Dienes, Z. (1969). Los Primeros Pasos en Matemática. Lógica y juegos lógicos. Barcelona: Teide .

Elliott, J. (1978). What is action-research in schools? . Journal of Curriculum Studies, 2.

Escamilla, A. (1992). Unidades didácticas, una propuesta de trabajo en el aula. Zaragoza: Colección Aula Reforma.

Fernández, J., \& Carrillo, J. (2014). Cómo se esfuerzan los alumnos en resolución de problemas matemáticos (I). Bolema: Boletim de Educação Matemática, 20. 
Galvéz, \& Pagés. (2016). La práctica de la enseñanza de la historia con base en los propósitos para enseñar: El caso de Mariana. Educação em Revista, 24.

Gómez, M. (2012). Didáctica de la matemática basada en el diseño curricular de educación inicial-nivel preescolar. León: Universidad de Léon.

Grupo Enciclopedia Catalana. (1978). Enciclopedia Catalana. Cataluña: Edicions 62.

Guzmán, W. (2012). Estrategias didácticas para potenciar el pensamiento variacional a través de situaciones problema, de los estudiantes del grado noveno de la institución educativa "San José del municipio de Betulia". Bogotá: Universidad Nacional de Colombia.

Huberman, \& Miles. (1994). Qualitative Data Analysis. California: Sage.

ICFES. (2016). Resumen Ejecutivo Colombia en PISA 2015. Bogotá: MinEducación.

ICFES. (2017). Informe Nacional de resultados Colombia en PISA 2015. Bogotá: MinEduación.

Kemmis, \& McTaggart. (1988). Cómo planificar la investigación-acción. Barcelona: Alertes.

Leal, L., \& Bong, S. (2015). La resolución de problemas matemáticos en los contextos de aprendizaje. Revista de Investigación de la Universidad Experimental del Libertador, 7193.

Lemus, C. (2015). Diseño de una propuesta didáctica para la enseñanza de métodos estadísticos descriptivos a través de experimentos. Medellín: Universidad Nacional de Colombia.

MEN. (2006). Estándares Básicos de Competencias en Lenguaje, Matemáticas, Ciencias y Ciudadanas. Bogotá: MinEducación.

MEN. (2011). Nivelemos 3 Matematicas-Guía docente. Bogotá: MinEducación.

MEN. (2016). Derechos Básicos de Aprendizaje V.2. Bogotá: Mineducación.

Montero, S. (2016). Los juegos tradicionales Kankuamos como estrategia para el desarrollo del pensamiento lógico-matemático. Valledupar: Universidad Popular del César.
Morales, M. \&. (2010). El impacto de la autoevaluación y el compromiso grupal en la mejora de actitudes ante el propio aprendizaje. Tecnológico de Monterey, 9. Obtenido de La docencia a través de la Investigación-Acción.

Moreno, A., \& Daza, B. (2014). Incidencia de estrategias metacognitivas en la resolución de problemas en el área de la matemática. Bogotá: Pontificia Universidad Javeriana .

Ordoñez, L. (2014). Estructuras Aditivas en la Resolución de Problemas Aditivos de enunciado verbal (PAEV). Palmira: Universidad Nacional de Colombia.

Piaget, J. (1977). The role of action in the development of thinking. New York: W. F. Overton y J. M. Gallager (Eds.).

Piñeiro, Pinto, \& Díaz. (2015). ¿Qué es la resolución de problemas? Revista Virtual Redipe, 9.

Polya, G. (1979). Cómo plantear y resolver problemas. México: Editorial Trillas.

Quineche, D. (19 de Junio de 2010). Red de Docentes de América Latina y el Caribe. Obtenido de PEDAGOGÍA COGNITIVA Y FORMACIÓN POR COMPETENCIAS: http://www.reddolac. org/profiles/blogs/pedagogia-cognitiva-y

Quintero, F., Resprepo, G., \& Padilla, N. (2016). La lúdica para el fortalecimiento de la resolución de problemas como competencia matemática en estudiantes de grado tercero de básica primaria. Bucaramanga: Universidad Cooperatuva de Colombia.

Rodríguez, \& Yáñez. (2012). Cambios actitudinales de los estudiantes inmersos en el proceso de enseñanza-aprendizaje ante la aplicación de diferentes actividades metodológicas innovadoras en las cátedras de ingés intensivo y computación de la Facultad de Ciencias y Humanidades. San Salvador: Universidad de El Salvador.

Rodriguez, Gil, \& García. (1996). Metodología de la investigación cualitativa. Madrid: Aljibe.

Rodríguez, L., Pimentel, L., \& Lozano, M. (2015). El método de proyecto para la formulación de problemas matemáticos. Atenas, 100-112. 
Rodríguez, M. (2014). Aprendizaje Centrado en el Alumno. México: UAEH (Universidad Autónoma del Estado de Hidalgo).

Rojas, A., Contreras, A., \& Árevalo, M. (2011). Intervención didáctica para promover el aprendizaje de las matemáticas, en niños con discalculalia. Repuestas, 9.

Schroeder, T., \& Lester, F. K. (1989). Developing understanding in mathematics via problem solving. N.C.T.M. En New directions for elementary school mathematics. Reston, Virginia.

Vergnaud. (1995). La Didactique a-t-elle un sens pour la formation des personnes peu motiveé? Migrants-formaction(100), 119-131.

Vidal, S. (1909). Aritmética. Madrid: Sucesores de Hernando. 